\title{
Pressure-induced isosymmetric phase transition in biurea
}

\author{
Craig L. Bull, Nicholas P. Funnell, Christopher J. Ridley, \\ Colin R. Pulham, Paul L. Coster, James P. Tellam \\ and William G. Marshall
}

\section{Published version information}

Citation: CL Bull et al. "Pressure-induced isosymmetric phase transition in biurea." CrystEngComm, vol. 21, no. 39 (2019): 5872-5881.

DOI: $10.1039 /$ C9CE01028A

This version is made available in accordance with publisher policies. Please cite only the published version using the reference above. This is the citation assigned by the publisher at the time of issuing the AAM. Please check the publisher's website for any updates. 


\title{
Pressure-Induced Isosymmetric Phase Transition in Biurea $^{\dagger}$
}

\author{
Craig L. Bull, ${ }^{a, *}$ Nicholas P. Funnell, ${ }^{a}$ Christopher J. Ridley, ${ }^{a}$ Colin R. Pulham, ${ }^{b}$ Paul L. \\ Coster, ${ }^{b}$ James P. Tellam, ${ }^{a}$ and William G. Marshall ${ }^{a}$
}

Received Date

Accepted Date

DOI: $00.0000 / x x x x x x x x x x$

\begin{abstract}
We report a pressure-induced transition to a new crystalline phase of biurea $\left(\mathrm{C}_{2} \mathrm{D}_{6} \mathrm{~N}_{4} \mathrm{O}_{2}\right)$. Neutron-powder diffraction and Raman spectra, measured up to $3.89 \mathrm{GPa}$ reveal this transition to be isosymmetric, where substantial intramolecular reorientation leads to an abrupt decrease in unit cell volume at $\sim 0.6 \mathrm{GPa}$ and an increase in some intermolecular contact distances. DFT and vibrational energy calculations suggest that the ambient pressure phase is stabilised by zero point energy and entropy, until the pressure-volume-driven transition at $0.6 \mathrm{GPa}$.
\end{abstract}

\section{Introduction}

The application of high pressure has been shown to access new phases of organic, solid-state materials, having been observed in alcohols? , amino acids? , bulky pharmaceuticals? , and even energetic materials. ? The importance of understanding, and controlling polymorphism, in such materials-particularly in the pharmaceutical industry-is now widely-appreciated. In molecular, organic crystals, the structures are often described in terms of hydrogen bonding motifs (when they are present). While it is recognised that hydrogen bonding is not always the most appropriate means for understanding the underlying forces that determine crystal structure, ? importance is still often ascribed to the hydrogen bond; it is thought to be structurally-significant, particularly in biological substances where it helps direct DNA base pairing and folds polypeptide chains into active proteins. Thus, the hydrogen bond is often used as a molecular recognition tool; directing assembly of co-crystal components, ? or targeting specific biological interactions. ? It forms a key consideration in the design process of orally-administered drugs, where half of the criteria in Lipinski's 'rule of five' concerns the quantity of hydrogen bond donors and acceptors.? Under pressure, the geometry of hydrogen bonds can often be altered, and this provides a strong, visual indication of the changes occurring in the crystal

\footnotetext{
a ISIS Neutron and Muon Facility, Rutherford Appleton Laboratory, Chilton, U. K.; Tel: +44(0)1235 445706; E-mail: craig.bull@stfc.ac.uk

${ }^{b}$ EastCHEM School of Chemistry, Joseph Black Building, David Brewster Road, Edinburgh, EH9 3FJ, UK

$\dagger$ Electronic Supplementary Information (ESI) available: CIFs for all refined structures, Rietveld fits for all diffraction data sets at pressure, refinement details for all refined data sets, variation of selected bond distances with pressure, high-pressure Raman spectra (hydrogenated and deuterated), hydrogenated INS, energy calculations for changing torsion angle, CCDC database queries, and void-space diagrams. CCDC 1935728-1935746
}

structure-for instance new bonds can be formed, and existing bonds broken, or symmetrised. ?

Urea $\left(\mathrm{CH}_{4} \mathrm{~N}_{2} \mathrm{O}\right)$ is a small molecule with enormous biological importance, being the end product in the removal of toxic ammonia from many organisms. It is comprised entirely of hydrogenbond donors and acceptors, and has been described as a model material for studying hydrogen bonding. ? ? Urea is known to exist in at least four solid phases; at ambient pressure it is tetragonal (phase I, $P 42_{1} m$ ) and transforms to an orthorhombic form (phase III, $P 2_{1} 2_{1} 2_{1}$ ) at $0.48 \mathrm{GPa}$. On further compression two additional orthorhombic structures are seen-phase IV $\left(P 2_{1} 2_{1} 2\right)$ at $2.8 \mathrm{GPa}$, and phase $\mathrm{V}$ at $\sim 7 \mathrm{GPa}$, which persists until at least $12 \mathrm{GPa}$ ? ? Across all phases, the variation in molecular arrangement is strongly manifested in the $\mathrm{N}-\mathrm{H} \cdots \mathrm{O}$ interactions, which have been identified as being key to its structural stability.

Given the variety of pressure-induced phases in urea, and the impact pressure has on hydrogen-bond geometry, it is interesting to look at a closely-related material, biurea $\left(\mathrm{C}_{2} \mathrm{H}_{6} \mathrm{~N}_{4} \mathrm{O}_{2}\right)$. It can be described as a pair of monomer urea units related to each other by a two-fold rotation perpendicular to the $\mathrm{N}-\mathrm{N}$ bond that connects the two urea molecules-see Figure 1. Thus, in phase I, the ambient pressure crystal structure (monoclinic, $C 2 / c$ ), a single urea molecule forms the asymmetric unit. ? The torsional freedom permitted about the $\mathrm{N}-\mathrm{N}$ bond means the molecule is non-planar, in contrast to its urea monomer, and now forms three crystallographically-distinct $\mathrm{N}-\mathrm{H} \cdots \mathrm{O}$ interactions (Figure 1 ).

Previous high-pressure investigations on biurea were performed using angle-dispersive X-ray diffraction (ADXRD) and Raman measurements in a diamond anvil cell (DAC). ${ }^{\text {Th }}$ The authors of this study showed that a transition occurs over the broad pressure range of $\sim 0.6-1.5 \mathrm{GPa}$, accompanied by substantive change in its hydrogen-bonded network. ? The new second phase (II) was proposed to be $P 2 / n$, and was found to revert to phase I 


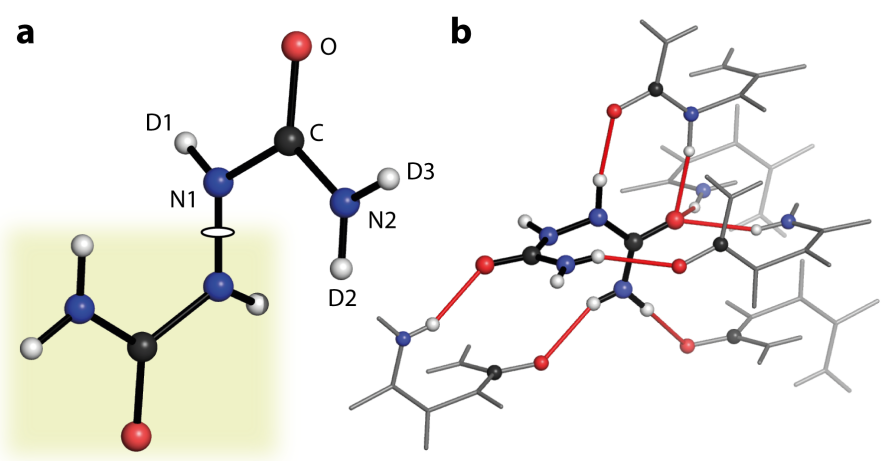

Fig. 1 a: Molecular structure of biurea $\left(\mathrm{C}_{2} \mathrm{H}_{6} \mathrm{~N}_{4} \mathrm{O}_{2}\right)$. The white oval bisecting the $\mathrm{N}-\mathrm{N}$ bond indicates the position of the 2-fold axis that relates the two urea units of the molecule to each other; the yellow box indicates one urea molecule-the asymmetric unit. b: Hydrogen bonding arrangement between biurea molecules in phase I. Hydrogen bonds are shown as solid red lines, and neighbouring molecules are shown with a wireframe representation, except for donor and acceptor $\mathrm{H}$-bonding atoms, which are shown as spheres. The labelling of the hydrogen positions with deuterium reflects the fact that we studied a perdeuterated sample here.

on decompression. However, it was not possible to determine the crystal structure in full and the authors suggested that the indexing of phase II was tentative. In this current study, we report high-pressure neutron-diffraction experiments performed on the perdeuterated biurea up to a pressure of $\sim 3.9 \mathrm{GPa}$, determining complete structural coordinates from higher-resolution powder data. We also report complementary high-pressure Raman and inelastic neutron spectroscopic (INS) measurements, along with DFT calculations, to provide an understanding of the driving forces behind the phase transition observed in biurea.

\section{Experimental}

\subsection{Preparation and characterisation of perdeuterated bi- urea}

To prepare perdeuterated biurea, a slurry of biurea- $\mathrm{h}_{6}(1.5 \mathrm{~g}$, TCI Chemicals) was heated at reflux in $\mathrm{D}_{2} \mathrm{O}(100 \mathrm{~mL})$ for 24 hours. The slurry was cooled and the $\mathrm{D}_{2} \mathrm{O}$ removed by freeze drying. This was repeated twice more to produce the fully perdeuterated material $(1.5 \mathrm{~g}, 95 \%)$. The resulting powder was placed in a $6 \mathrm{~mm}$ vanadium canister and a time-of-flight (TOF) diffraction pattern measured on the PEARL diffractometer at the ISIS Neutron and Muon Source in the UK ${ }^{\text {? }}$. Rietveld refinement of the powder pattern permitted determination of the level of deuteration of the sample, and was performed using the GSAS suite of programmes? ? The H/D site occupancy was allowed to freely refine at each site, resulting in $100 \%$ occupation by deuterium. A diffraction pattern of the hydrogenated sample (used as purchased) was also obtained in the same way, but the diffraction pattern suffered from significant incoherent scattering, limiting the ability to perform reliable Rietveld refinement of the hydrogenated material (see S.I. ${ }^{\dagger}$ ).

\subsection{High-pressure neutron diffraction}

High-pressure TOF neutron-powder diffraction measurements were conducted on the PEARL high-pressure diffractometer. ${ }^{?}$ The perdeuterated biurea powder was loaded into a null-scattering Ti-Zr alloy capsule gasket, ? which was then sealed within a V3 Paris-Edinburgh (PE) press, ? equipped with single-toroidal zirconia-toughened alumina (ZTA) anvils. In addition to the powdered sample, a small lead pressure marker ( $\sim 1 \mathrm{~mm}$ diameter) and a mixture of perdeuterated methanol:ethanol (4:1 by volume) were added to the gasket, the latter acting as a hydrostatic pressure transmitting fluid. ${ }^{\text {? }}$ A series of diffraction patterns were collected, with increasing hydrostatic pressure, up to a maximum 3.89 GPa. During the experiment, the unit-cell volume of the $\mathrm{Pb}$ pressure calibrant was used to determine the pressure from a Murnaghan equation of state (EoS). ${ }^{?}$ TOF data were normalised and corrected using in-house software. ' A beamline-developed correction for the wavelength and scattering-angle dependence of the neutron attenuation by the ZTA anvils and Ti-Zr gasket materials was applied to the observed pattern. ' GSAS and EXPGUI were used for Rietveld refinement. ? ? In order to minimise correlation between parameters arising from the non-orthogonal axes of the $C 2 / c$ cell, we used the alternative setting $I 2 / a$ which had a $\beta$ angle closer to $90^{\circ}$. The scattering from the $\mathrm{Pb}$ and ZTA materials were accounted for by additional crystalline phases in the Rietveld refinements. A least-squares fit to the volume, as a function of pressure, was used to determine the Vinet EoS. ?

\subsection{High-pressure Raman spectroscopy}

Ambient and high-pressure Raman spectra were measured for both the hydrogenated and perdeuterated biurea samples. Spectra were recorded in backscattering geometry using an in-house system comprised of a Princeton Instruments SP2500i with $1800 \mathrm{~g}$ holographic and $1200 \mathrm{~g}$ blaze gratings. Two Optigrate super notch filters are incorporated into the system, and a diode laser $(\lambda=532 \mathrm{~nm})$ was focussed using a $20 \times$ Mitutoyu objective lens, with a laser power of $5 \mathrm{~mW}$ at the sample position. All spectra were recorded at room temperature. High-pressure Raman spectroscopy was carried out in an Almax Nitro membranedriven DAC equipped with $500 \mu \mathrm{m}$ culet diamonds, and a $250 \mu \mathrm{m}$ thick copper beryllium gasket, indented to $90 \mu \mathrm{m}$ thickness, with a $200 \mu \mathrm{m}$ diameter hole forming the sample chamber. A ruby sphere was included in the sample chamber to act as a pressure calibrant, using the fluorescence method. ? For measurements on hydrogenated biurea, a hydrogenated methanol:ethanol (4:1) solution was used a pressure-transmitting fluid, and a perdeuterated fluid was used for the perdeuterated biurea sample, to avoid unwanted H/D exchange.

\subsection{Inelastic neutron scattering measurements}

Inelastic neutron scattering measurements (INS) were performed on the TOSCA instrument at the ISIS Neutron and Muon facility, UK. ? Perhydrogenated (1.3806 g) and perdeuterated (1.1544 g), powdered, biurea samples were loaded into $\mathrm{Al}$ pouches and spectra recorded for each sample at $20 \mathrm{~K}$. 


\subsection{Lattice, intra- and inter-molecular, and vibrational cal-} culations

Geometry optimisations were performed using CASTEP 7.0.3, where starting coordinates were taken from Rietveld-refined val-

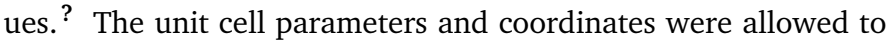
optimise while using space group symmetry. The PBE exchangecorrelation functional was used, with the TS dispersion correction scheme, along with on-the-fly pseudopotentials.? ? Convergence testing of the plane wave cutoff energies and $k$-point grid spacing gave optimal values of $950 \mathrm{eV}$ and $0.08 \AA$, respectively. The convergence criteria were set as: total energy per atom, $5 \times 10^{6} \mathrm{eV}^{-3}$; maximum force, $0.001 \mathrm{eV} \AA^{-3}$; maximum displacement, $5 \times 10^{-4} \AA$; and maximum stress of $0.02 \mathrm{GPa}$. Phonon calculations were performed using the finite displacement method, with a phonon energy tolerance of $1 \times 10^{5} \mathrm{eV}$ and a displacement amplitude of $5.4 \times 10^{3} \AA$. INS spectra were calculated from the resulting vibrational modes using the AbINS module in Mantid.?

The energy change resulting from molecular conformation was calculated by performing fixed-coordinate gas-phase calculations, using the CASTEP-optimised molecules, in Gaussian09 at the MP2/6-31G level of theory.?

\section{Results}

\subsection{Reassigning the phase II structure}

Figure 2 shows the neutron-powder diffraction pattern of perdeuterated biurea at $0.01 \mathrm{GPa}$. The Rietveld profile refinement has been performed using the previously-reported monoclinic structure (in its $I 2 / a$ setting) as a starting point and the resulting atomic coordinates are in agreement with those reported in Ref ? ; details of all structural refinements are available in the S.I. ${ }^{\dagger}$ The powder patterns show that no significant changes are observed up to $\sim 0.5 \mathrm{GPa}$, other than the expected unit cell volume decrease (Figure 2). At $0.62 \mathrm{GPa}$, clear differences in the pattern indicate the phase transition which, in our neutron experiment, occurs over a single pressure step. This stands in contrast to the previous X-ray diffraction study of hydrogenated biurea, where the transition to phase II took place over the range 0.6$1.5 \mathrm{GPa}$ ?

We attempted to fit the data with the $P 2 / n$ model, proposed in Ref. ? however we were unsuccessful; we were never able to account for all sample peaks simultaneously. Instead we found that the cell symmetry was still consistent with that of phase I $C 2 / c$ (reported as $I 2 / a$ here) but accompanied by a $\sim 5 \%$ unit cell volume collapse-see Table 1. The Rietveld fit shown in Figure 2 for phase II uses the $I 2 / a$ cell. With further increase in pressure, the diffraction pattern shows no additional changes beyond general cell volume reduction up to $3.89 \mathrm{GPa}$ (Figure 2). If the sample has undergone the same transition as that observed in Ref ? then we suggest that the previous phase II indexing may be incorrect, possibly because of the limited resolution of the high-pressure X-ray data. Similarly to the previous high-pressure study, we observe upon decompression to ambient pressure transformation of the sample back to phase I.
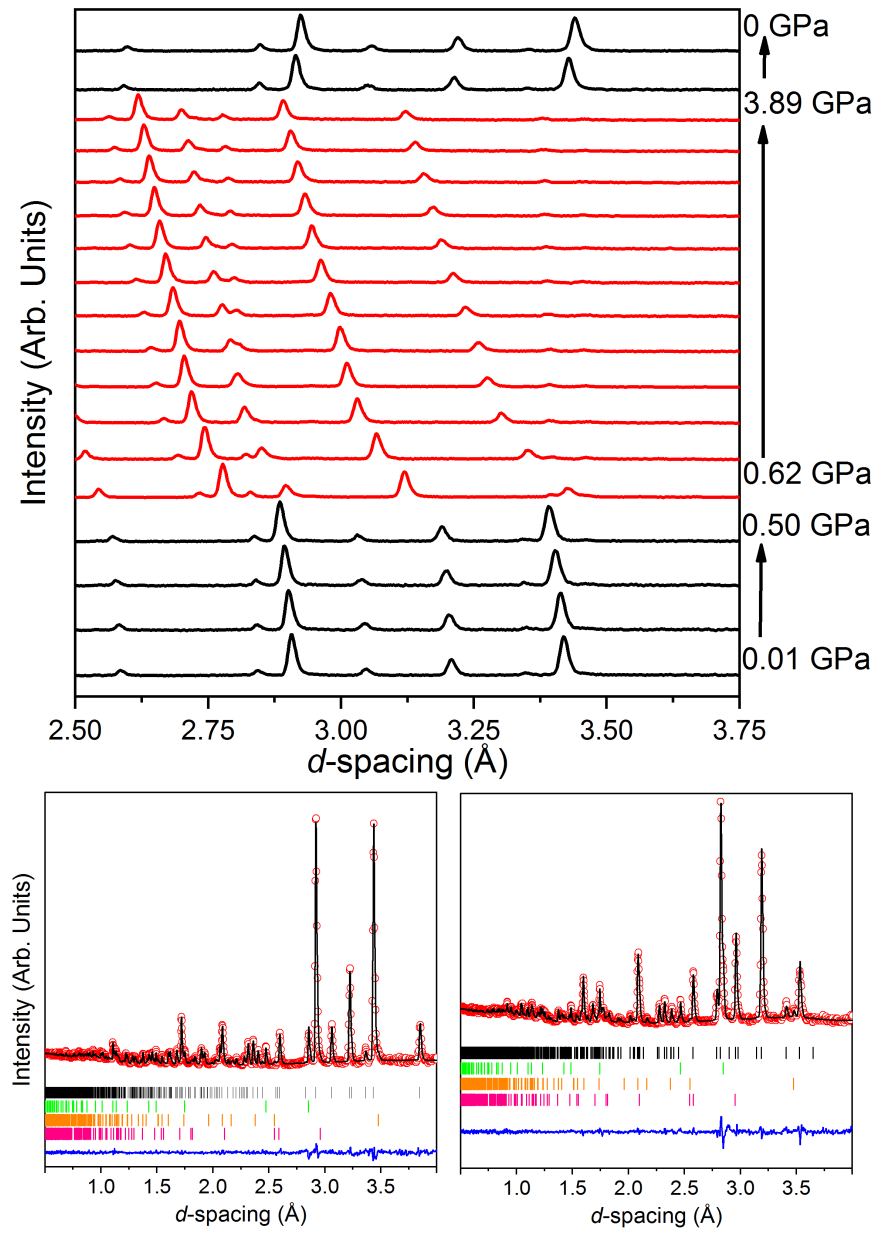

Fig. 2 Top: powder diffraction patterns of biurea with increasing pressure to $3.89 \mathrm{GPa}$ and subsequent recovery to phase I on decompression (black and red traces indicate phase I and II, respectively). Lower panels: representative Rietveld fits to data, collected at $0.01 \mathrm{GPa}$ (left) and $0.62 \mathrm{GPa}$ (right). In both lower panels data are shown as open red circles, the calculated profile in black, and the residual in blue. The tick marks show the reflection positions for each phase: (from top to bottom) biurea (black); $\mathrm{Pb}$ (green); $\mathrm{Al}_{2} \mathrm{O}_{3}$ (orange); and $\mathrm{ZrO}_{2}$ (pink). The results of all Rietveld refinements described in this text can be found in the S.I. ${ }^{\dagger}$ 
Table 1 Refinement details for select high-pressure data collections. A table containing refinement details of all high-pressure data can be found in S.I. ${ }^{\dagger}$

\begin{tabular}{lllll}
\hline Pressure $(\mathrm{GPa})$ & 0.01 & 0.50 & 0.62 & 3.89 \\
\hline Phase & $\mathrm{I}$ & $\mathrm{I}$ & $\mathrm{II}$ & $\mathrm{II}$ \\
Chemical formula & $\mathrm{C}_{2} \mathrm{D}_{6} \mathrm{~N}_{4} \mathrm{O}_{2}$ & $\mathrm{C}_{2} \mathrm{D}_{6} \mathrm{~N}_{4} \mathrm{O}_{2}$ & $\mathrm{C}_{2} \mathrm{D}_{6} \mathrm{~N}_{4} \mathrm{O}_{2}$ & $\mathrm{C}_{2} \mathrm{D}_{6} \mathrm{~N}_{4} \mathrm{O}_{2}$ \\
Formula weight $\left(\mathrm{g} \mathrm{mol}^{-1}\right)$ & 124.13 & 124.13 & 124.13 & 124.13 \\
Crystal system & Monoclinic $(I 2 / a)$ & Monoclinic $(I 2 / a)$ & Monoclinic $(I 2 / a)$ & Monoclinic $(I 2 / a)$ \\
$a$-axis $(\AA)$ & $9.3308(8)$ & $9.3429(5)$ & $9.4872(3)$ & $9.3926(5)$ \\
$b$-axis $(\AA)$ & $4.6415(4)$ & $4.5550(2)$ & $3.8140(2)$ & $3.3328(1)$ \\
$c$-axis $(\AA)$ & $11.4722(10)$ & $11.3567(5)$ & $12.8589(10)$ & $13.1269(7)$ \\
$\beta\left(^{\circ}\right)$ & $82.1069(6)$ & $82.183(5)$ & $77.936(7)$ & $77.329(5)$ \\
Volume $\left(\AA^{3}\right)$ & $492.10(10)$ & $478.82(3)$ & $455.01(4)$ & $400.92(2)$ \\
Density $\left(\mathrm{g} \mathrm{cm}^{-3}\right)$ & 1.68 & 1.72 & 1.81 & 2.06 \\
$R 1 / R_{\text {wp }}$ & $4.0 / 3.5$ & $3.3 / 2.9$ & $3.8 / 3.4$ & $3.5 / 3.0$ \\
Goodness of fit $\chi^{2}$ & 1.3 & 1.1 & 1.5 & 1.0 \\
\hline
\end{tabular}

Such isosymmetric structural phase transitions must be accompanied by a volume discontinuity and so are necessarily first order in nature. ' Temperature and pressure-driven examples are known, including the II-IV transition in $\mathrm{KNO}_{3}$ at $\sim 0.3 \mathrm{GPa}$ accompanied by a $11.5 \%$ volume change, ${ }^{?}$ the framework collapse of $\mathrm{KTiOPO}_{4}$ at $5.5 \mathrm{GPa}$ with a volume decrease of $4.2 \%$, ? and in octafluoronaphthalene at $\sim 267 \mathrm{~K}$ and $\sim 0.8 \mathrm{GPa} .{ }^{\text {? }}$ ?

\subsection{Compression of the biurea phases}

Compression behaviour can only be understood directly from unit cell dimensions when they are orthogonal, as they lie coincident with the principal axes of the strain tensor. For non-orthogonal axes (as is the case here), these must be reformulated into a Cartesian set. We have determined the principal axes (and their relation to the unit-cell axes), and their corresponding compressibilities, ? for both biurea phases, shown in Figure 3 a \& b, and detailed in Table 2 In particular, for phase I, negative linear compressibility (NLC) along the $\mathbf{X}_{3}$ direction, of $-2.7(5) \mathrm{TPa}^{-1}$ is notable. This behaviour persists to phase II, albeit with a reduced magnitude, $-0.856(2) \mathrm{TPa}^{-1}$. This NLC in both phase I and II can be attributed to intramolecular conformational changes in the molecule where the dihedral angle $(\theta)$ between the mean planes through the non-hydrogen atoms of the biurea monomers is seen to flatten on compression (Figure 3 c\& d), similar to the hinging mechanisms seen in other molecular 'wine-rack' materials which exhibit NLC behaviour. ' Such NLC behaviour in organic materials was thought to be rare, however, there are an increasing number of examples of organic materials exhibiting such behaviour with examples including methanol monohydrate $\left(-3.8 \mathrm{TPa}^{-1}\right)^{\text {? }}$, ammonium oxalate monohydrate $\left(-2.3 \mathrm{TPa}^{-1}\right)^{?}$ and the Y polymorph of ROY $\left(-5.5 \mathrm{TPa}^{-1}\right) ?$ with similar values of NLC.

Figure 4 shows the fitted Vinet EoS to the phase II unit cell variation with pressure-numerical details are given in Table 2. Given the limited number of data points, as a result of the narrow pressure range over which phase I is stable, we were unable to determine its bulk modulus derivative $B^{\prime}$ reliably, thus it was held fixed at the value determined for phase II. Unusually, the bulk moduli appear to indicate that the denser phase II $\left(B_{0}=6.7(11) \mathrm{GPa}\right)$ is more compressible than phase I $\left(B_{0}=15.4(18) \mathrm{GPa}\right)$, this unex- pected result may well be a result that the phase space of I is poorly sampled. These values are broadly consistent with the bulk moduli measured for the urea phases-tetragonal phase I has $B_{0}=9.9(4) \mathrm{GPa}, B^{\prime}=12.4(17)$, and orthorhombic phase III has $B_{0}=9.9(5) \mathrm{GPa}, B^{\prime}=7.1(17),{ }^{?}$ but other studies find rather different values for phase III; $B_{0}=19.4 \mathrm{GPa}, B^{\prime}=4.5$ ? $^{\text {Th }}$ The determined values of biurea are also not unusual for organic materials for example, glycolide has $B_{0}=6.6(4) \mathrm{GPa}$, ? solid ethanol $B_{0}=9.9(5) \mathrm{GPa}, ?$ and the Y polymorph of ROY $B_{0}=9.9(5) \mathrm{GPa} . ?$

\subsection{Effect on hydrogen bonding}

The need to reduce cell volume as pressure increases will necessarily increase the density of molecular packing. This will be reflected in intermolecular contacts, particularly in a material that is dominated by hydrogen bonding. There are three distinct bonds-we consider first the D3... O interaction (see Figure 1), the length of which decreases in linear fashion over the whole pressure range studied-see S.I. ${ }^{\dagger}$ for plots. The D2 $\cdots$ O bond shows a more pronounced change on increasing pressure and particularly near the region of the phase transition. However, the $\mathrm{D} 1$... O interaction length shows more anomalous behaviour; after initially decreasing with pressure to $1.79 \AA$-approaching the lower limit for N-H/D ... O contacts in the Cambridge Structural Database? $^{\text {? }}$ (see S.I. ${ }^{\dagger}$ )—it increases to a maximum of $1.86 \AA$ on transformation to phase II, before smoothly decreasing again on further compression.

The extent of deuterium atom (de)localisation in the hydrogen bonds located on the secondary amines will, to some extent, be reflected in the $\mathrm{N}-\mathrm{N}$ bond distance-suggestive of the degree of single/double bond character-as H-bond contact distances change with pressure. At ambient pressure the $\mathrm{N}-\mathrm{N}$ bond distance is $1.38 \AA$ lying somewhere between the values expected for a single $(\sim 1.47 \AA)$ and double $(\sim 1.24 \AA)$ bond. The N-N bond length increases, up to $1.39 \AA$ as the phase transition is approached, showing increased localisation of the deuterium atoms on the amides, before reversing trend in phase II and decreasing linearly to $1.34 \AA$ by $3.89 \mathrm{GPa}$-indicating delocalisation of the deuterium atoms between molecules.

The behaviour of the $\mathrm{N}-\mathrm{H}(\mathrm{D})$ stretching modes, spanning 

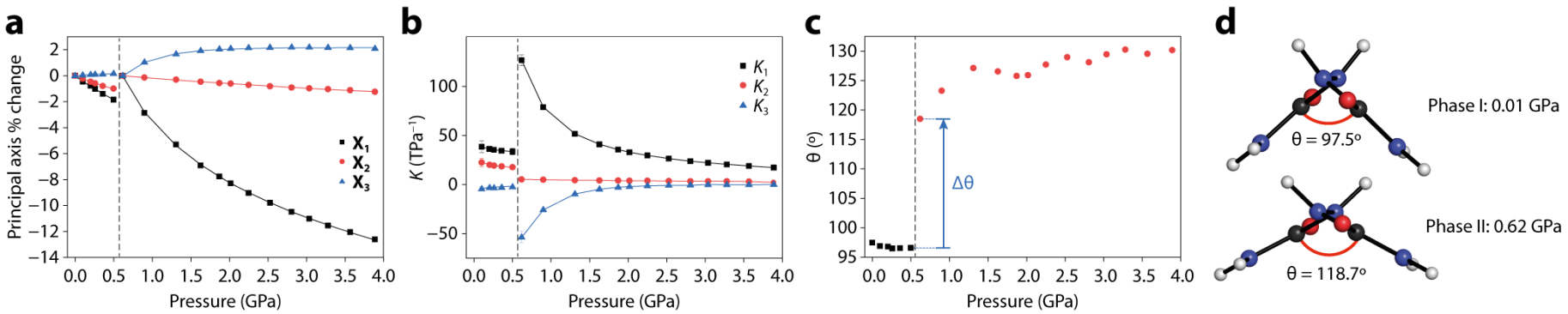

Fig. 3 a: Relative change in length of the principal directions in phase I and II, as a function of pressure. The vertical dashed line indicates the phase boundary. $\mathbf{X}_{1}$ is shown by black squares, $\mathbf{X}_{2}$ by red circles and $\mathbf{X}_{3}$ with blue triangles (see main text and Table 1 for further details). b: principal linear compressibilties of phase I and II of biurea: $K_{1}$ is shown by black squares, $K_{2}$ by red circles and $K_{3}$ with blue triangles. c: The change in dihedral angle $(\theta)$ between mean planes of the biurea monomers, on compression. $\mathrm{d}$ : the dihedral angle $(\theta)$ discontinuity at the transition illustrated on the biurea molecule, shown in projection within the planes of the $\mathrm{C}, \mathrm{N}$, and $\mathrm{O}$ atoms of each biurea unit (approximately aligned with the $\mathrm{N}-\mathrm{N}$ bond).

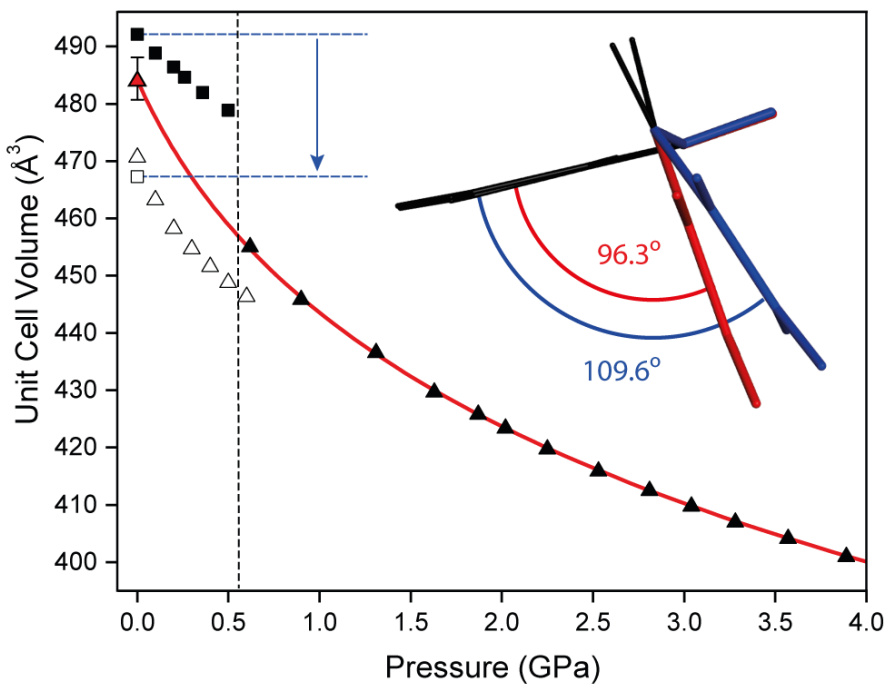

Fig. 4 Variable-pressure experimental ( $\square$-phase I and $\mathbf{\Delta}$-phase II) and DFT-optimised ( $\square$-phase I and $\triangle$-phase II) unit-cell volumes. In all experimental data error bars are smaller than the symbols. The vertical dashed line indicates the experimentally-observed I-II phase boundary. The solid red line depicts the Vinet EoS for phase II; the red triangle indicates the refined $V_{0}$ parameter by the EoS. The cell volume of phase I, when optimised (open square), closely matches that of phase II, hence the requirement to keep its unit cell fixed. The downward, blue arrow indicates the zero-pressure volume difference between experiment and DFT optimisation of phase I. The inset shows DFT-optimised molecules at ambient pressure for phase I (red, fixed cell) and II (blue) in projection in the mean planes of the individual biurea units. One half of the molecule is overlaid for both phases, and coloured black.

$\sim 2300-2400$ rel cm $^{-1}$ in the Raman spectra with increasing pressure are shown the S.I. ${ }^{\dagger}$ Due to the finite resolution of the spectrometer, the spectra provide insight into the average vibrational behaviour of the three hydrogen bonds discussed previously. These shows that the vibrational modes soften upon pressure increase, without any significant discontinuity through the phase transition. However, our high-pressure Raman spectroscopy measurements confirm the behaviour seen crystallographically: stiffening of the $\mathrm{C}-\mathrm{N}$ stretching mode and $\mathrm{C}-\mathrm{N}-\mathrm{N}$ in-plane bend (in the region of $1000 \mathrm{rel} \mathrm{cm}^{-1}$ - see Figure 5 a) is consistent with increasing $\mathrm{N}-\mathrm{N}$ double-bond character, as hydrogen bond lengths
Table 2 Bulk moduli $B_{0}$, associated derivatives $B^{\prime}$, zero-pressure volume $V_{0}$, and median principal compressibilities $K_{\mathrm{i}}$ of phase I and II of biurea. Also given are the principal axes $\mathbf{X}_{\mathrm{i}}$ and their relationship to the unit-cell axes. The value of $B^{\prime}$ for phase I was fixed to the value of phase II for the determination of $B_{0}$ and $V_{0}$.

\begin{tabular}{llll}
\hline Phase & $V_{0}\left(\AA^{3}\right)$ & $B_{0}(\mathrm{GPa})$ & $B^{\prime}$ \\
\hline I & $492(1)$ & $15.4(18)$ & 12.3 \\
II & $484(4)$ & $6.7(11)$ & $12.3(8)$ \\
\hline & Principal axes & $K_{i}\left(\mathrm{TPa}^{-1}\right)$ & Axis direction \\
\hline I & $\mathbf{X}_{1}$ & $34.6(18)$ & $=\mathbf{b}$ \\
& $\mathbf{X}_{2}$ & $18.5(13)$ & $\approx-0.05 \mathbf{a}+\mathbf{c}$ \\
& $\mathbf{X}_{3}$ & $-2.7(5)$ & $\approx \mathbf{a}-0.14 \mathbf{c}$ \\
II & $\mathbf{X}_{1}$ & $26.25(19)$ & $=-\mathbf{b}$ \\
& $\mathbf{X}_{2}$ & $3.4834(5)$ & $\approx-\mathbf{a}+0.21 \mathbf{c}$ \\
& $\mathbf{X}_{3}$ & $-0.856(2)$ & $\approx 0.13 \mathbf{a}+\mathbf{c}$ \\
\hline
\end{tabular}

decrease (our mode assignments are informed by those of ref ? ). The sudden discontinuity in intensity of the $\mathrm{C}-\mathrm{N}-\mathrm{N}$ mode relative to the $\mathrm{C}-\mathrm{N}$ stretch is indicative of a change in polarisability (Fig 5b), which mirrors the variation in dihedral angle $(\theta)$ over the transition (as in Fig 3). For the hydrogenated sample, Figure $5 \mathrm{p}$ shows the relative intensity with increasing pressure of the $\mathrm{C}-\mathrm{N}$ stretching and $\mathrm{C}-\mathrm{N}-\mathrm{N}$ in-plane bending mode around $1000 \mathrm{rel} \mathrm{cm}^{-1}$, and shows discontinuous behaviour over a narrow pressure range.

\section{Discussion}

\subsection{Why does phase I exist?}

The I $\rightarrow$ II transformation is indicated by an abrupt volume drop (see Figure 4), intramolecular deformation (Figure 3), and the increase in length of the D1... O H-bond. However, the two phases have the same topology; space group symmetry is unchanged, as is the molecular packing arrangement. In general, crystal structures prefer efficient packing-density is often used as a scoring metric in crystal structure prediction, favouring one candidate structure over another-so, on a geometric basis, it is not entirely clear why phase I should exist at all, as opposed to some form of the phase II structure extrapolated to ambient pressure.

There is a significant volume change in the material on compression from phase I to II (Figure 4). However, this is accom- 

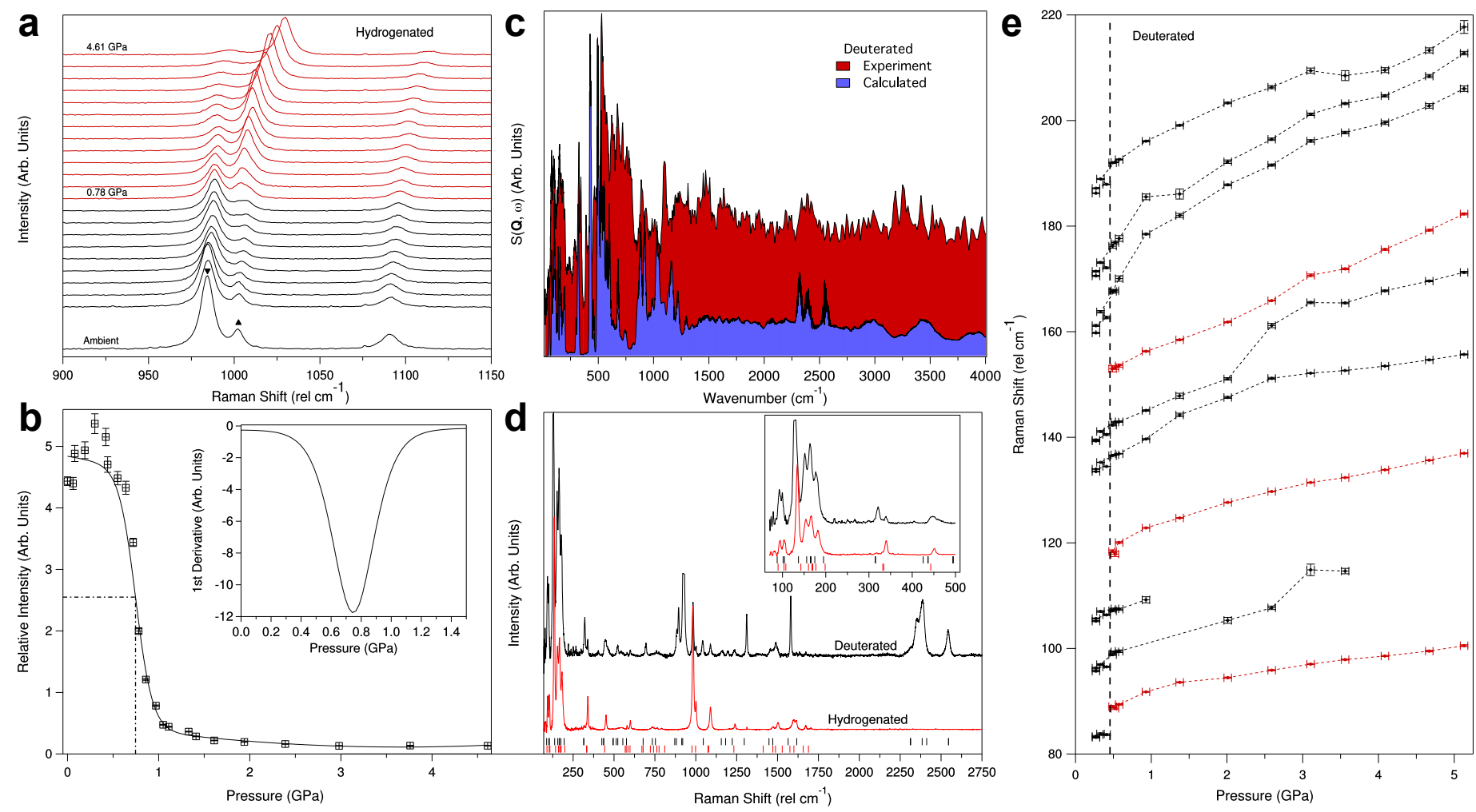

Fig. 5 Vibrational spectra of biurea. a: Variation in Raman spectra of perhydrogenated biurea in the region around the $\mathrm{C}-\mathrm{N}$ stretching $(\mathbf{\nabla})$ and $\mathrm{C}-\mathrm{N}-\mathrm{N}$ in-plane bending $(\boldsymbol{\Lambda})$ vibrational modes with increasing hydrostatic pressure (phase I indicated by black spectra and phase II by red). b: Relative intensity of the $\mathrm{C}-\mathrm{N}$ stretching and $\mathrm{C}-\mathrm{N}-\mathrm{N}$ in-plane bending modes between as a function of pressure for the hydrogenated sample (inset shows first derivative of fit to trend, used to determine the transition pressure). c: Experimental (red) and theoretical (blue) INS spectra of perdueterated biurea. d: Experimental Raman spectra of perdeuterated (top) and perhydrogenated (bottom) biurea at ambient pressure (outwith of the DAC). The vertical tick marks show the expected position of the vibrational modes as predicted by periodic DFT calculations (this study), black perdeuterated and red perhydrogenated. e: External vibrational mode shift of perdeuterated biurea with increasing pressure. Further high-pressure Raman spectra can be found in the S.I. ${ }^{\dagger}$, as can the experimental and theoretical INS spectra for perhydrogenated biurea.

panied by only a relatively subtle change in molecular packing of the biurea molecule within the lattice. It is therefore, interesting to try and understand, why phase I is the more stable form under ambient conditions, and rationalise this in terms of their relative Gibbs free energies.

\subsection{Density Function Theory geometry optimisations}

To compare the relative energies of the two polymorphs under ambient pressure conditions, it was necessary to first produce a hypothetical phase II structure, which we obtained via planewave DFT calculations. A geometry-optimised phase I structure was also generated for comparison. The ambient-pressure phase II structure started with the cell parameters and coordinates of the phase II model at $0.6 \mathrm{GPa}-$ the lowest pressure for which experimental data were available. Allowing the cell parameters and the coordinates to optimise resulted in a cell volume of $470.6 \AA^{3}$; approximately $14 \AA^{3}$ below the volume of the fitted the Vinet EoS (484(4) $\AA^{3}$ ). Additional phase II optimisations were performed between ambient pressure and $0.6 \mathrm{GPa}$, which all used the same starting structure, but the pressure was varied in $0.1 \mathrm{GPa}$ increments. The resulting pressure-volume trend is very similar to that predicted by the EoS fit to experimental data, albeit with a constant volume offset. Experimental and DFT cell volume de- tails are given in Figure 4, along with the fitted Vinet EoS to the experimental phase II data, and a comparison of the optimised molecular geometries at ambient pressure.

Initial geometry optimisation of phase I used the experimentally-determined ambient crystal structure as a starting model, and permitted the lattice parameters and atomic coordinates to vary. However, this led to a large volume reduction (see Figure 4), where the resulting structure converged on essentially the same endpoint as the phase II optimisation, using the strategy detailed above. Thus the decision was made to hold the lattice parameters fixed to experimental values, for phase I, and allow only the atomic positions to optimise-this structure was used for subsequent energy calculations. The reliability of the DFT-optimised structures was assessed by calculating INS spectra at the $\Gamma$ point, and comparing against those collected experimentally on TOSCA. Figure 5 - shows a comparison of the as-measured INS spectra and those calculated for the perdeuterated phase I of biurea at ambient pressure-there is generally favourable agreement between experiment and theory (see S.I. for experimental, and calculated, INS spectra of the perhydrogenated sample ${ }^{\dagger}$ ).

The most pronounced difference between the optimised, ambient-pressure, phase I and II structures is intramolecular: a 
large change in the angle, $\theta$ relating the two monomer units. In concordance with Ref. ? , we calculate $\theta$ as the angle of inclination where mean planes containing the $\mathrm{C}, \mathrm{N}$, and $\mathrm{O}$ atoms that make up each biurea monomer, intersect. These angles are $96.26^{\circ}$ in phase I and $109.63^{\circ}$ in phase II; the difference between these, $13.37^{\circ}$, is smaller than the difference between experimental phase I and II structures, either side of the phase transition$22.03^{\circ}$.

\subsection{Thermodynamic stabilities}

First, differences attributed to molecular conformation alone were evaluated by performing ab-initio, single-point energy calculations in Gaussian, at the MP2 level, on a series of gas-phase molecules. ' Each molecule was fixed to have identical geometry (informed by the ambient-pressure crystal structure), except for the flexible torsion angle, which was systematically varied in steps of $5^{\circ}$ between 40 and $180^{\circ}$. The calculated energies are plotted in the S.I. ${ }^{\dagger}$, and show that there are no abrupt discontinuitiesthe energy varies smoothly as a function of angle. This suggests that it is not the case that the molecule is attempting to avoid particular conformations that cause instability. However, a direct comparison of the Gaussian single-point energies, using the DFToptimised coordinates for the two phases at ambient pressure, does favour phase I by $16.3 \mathrm{kJmol}^{-1}$ (Table 3).

Next, a comparison of the dispersion-corrected DFT (DFT+D) energies, which are sensitive to both inter- and intra-molecular geometry, shows phase II to be more stable by $1.25 \mathrm{kJmol}^{-1}$ per molecule. We note that the DFT $+\mathrm{D}$ and Gaussian energies are not directly comparable, due to the differing approaches by which they have been calculated, but if it is the case that intramolecular geometry truly favours phase I, then the DFT results indicate that this is outweighed by lattice energy, stabilising phase II.

What ultimately dictates the thermodynamic stability of a material is the collective contributions of enthalpy $H$, and entropy terms $T S$, toward its Gibbs free energy; $G=H-T S$. The enthalpy term can be decomposed further to a sum of internal energy $U_{0}$, pressure $\times$ volume $p V$, zero point energy ZPE, and vibrational contributions $E_{\mathrm{vib}}$. Vibrational modes are not normally calculated as part of a DFT geometry optimisation, however as ZPE, $E_{\text {vib }}$ and entropic energy contributions depend on vibrational modes, as described by Rivera et al, ? we calculate these terms at $295 \mathrm{~K}$; all energy terms are given in Table 3 . We omit $p V$ contributions as these are negligible at ambient pressure, and can be ignored. Our calculated values show that although the total DFT $+\mathrm{D}$ energy favours phase II by $1.25 \mathrm{kJmol}^{-1}$, the $\mathrm{ZPE}$-which is most strongly influenced by the large-frequency vibrations of hydrogen bonding-finds phase I to be more stable by $0.95 \mathrm{kJmol}^{-1}$. Similarly the $T S$ contribution, (which becomes increasingly important at higher temperatures) shows a preference for phase I of $1.84 \mathrm{kJmol}^{-1}$. The $E_{\mathrm{vib}}$ contribution to $H$ favours phase II, although it is smaller in magnitude; $0.55 \mathrm{kJmol}^{-1}$. Taken altogether, we can calculate a corrected Gibbs free energy, which finds phase I to be the more stable by $0.97 \mathrm{kJmol}^{-1}$.

While these calculations are performed within the limitations of the DFT models we describe here, this small energy difference
Table 3 Calculated energy contributions to phase stability; $U_{\text {conf }}, \mathrm{DFT}+\mathrm{D}$, $\mathrm{ZPE}, E_{\mathrm{vib}}$, and $T S . G=(\mathrm{DFT}+\mathrm{D})+\mathrm{ZPE}+E_{\mathrm{vib}}-T S . E_{\mathrm{vib}}$ and $T S$ are calculated using a temperature of $295 \mathrm{~K}$. All energies are quoted per molecule in units of $\mathrm{kJmol}^{-1}$

\begin{tabular}{lrrr}
\hline Phase & I & II & I-II \\
\hline$U_{\text {conf }}$ & -1176384.45 & -1176368.12 & -16.33 \\
DFT+D & -231497.50 & -231498.75 & +1.25 \\
ZPE & 230.74 & 231.69 & -0.95 \\
$E_{\text {vib }}$ & 23.19 & 22.64 & +0.55 \\
$T S$ & 42.57 & 40.73 & +1.84 \\
$G$ & -231286.13 & -231285.16 & -0.97 \\
\hline
\end{tabular}

separating the two polymorphs is typical; energies of just a few $\mathrm{kJmol}^{-1}$ between different crystalline forms of molecular materials are common. ${ }^{\text {? }}$ The denser phase II is favoured as a static structure, but only by considering the ambient-temperature vibrational characteristics of this heavily hydrogen-bonded crystal can we recreate the relative stabilities that are observed experimentally. Performing the same calculations at $0.5 \mathrm{GPa}$, using experimental and optimised lattice parameters for phases I and II, respectively, resulted in the same phase preference from each individual energy contribution, but all I-II differences were reduced in magnitude. Above ambient pressure, the $p V$ contribution becomes significant, and we estimate it to favour phase II by $2.25 \mathrm{kJmol}^{-1}$ at $0.5 \mathrm{GPa}$, which changes $G$ sufficiently to favour phase II overall by $1.09 \mathrm{kJmol}^{-1}$. However, there are significant volume differences between our DFT models, which will overtly influence the $p V$ term. When estimating a more realistic volume for phase II from our EoS (see Figure 4), then the $p V$ reward for phase II is $1.52 \mathrm{kJmol}^{-1}$, leading to a volume corrected $\Delta G_{\mathrm{I}-\mathrm{II}}$ of $0.36 \mathrm{kJmol}^{-1}$-its near-zero value being consistent with its proximity to the I $\rightarrow$ II transition pressure.

\subsection{Raman and INS spectroscopy}

The previous high-pressure study on biurea by Yan et al, suggested that the $\mathrm{I} \rightarrow$ II transition in biurea was quite sluggish, with signatures of the transition being observed over pressures between 0.6-1.5 GPa, using powder X-ray diffraction and Raman spectroscopy. This stands in contrast to the rather more sharp transition we see here. However, there are two key differences in our experiments from which this discrepancy might arise; i) experiment timescales and; ii) the role of deuteration. We note that a common third difference might be choice of pressure-transmitting medium. However, in this case we also use a methanol-ethanol mixture, as in Ref. ? , where the authors already ruled out the possibility of any medium-related effects, noting identical behaviour when using either methanol-ethanol or nitrogen.

The highly-penetrating nature of neutrons means sample count times are generally much greater that the equivalent X-ray experiment, leading to slower compression of the sample in the former. The rate of pressurisation is known to influence transition pressures, ? ? and in some cases can kinetically trap a phase if the pressure change is sufficiently rapid.? Our sample was held at each pressure point for approximately 1 hour, which may 
have allowed it enough time to relax at $0.6 \mathrm{GPa}$ and transform completely - it is likely that the DAC experiment in Ref. ? would have proceeded rather more quickly. In the case of deuteration, the isoelectronic, yet heavier deuterium atom is known to alter phase boundaries, ? ? and in some rare instances, lead to socalled isotopic polymorphism. ? ? ? To investigate both these possibilities, we measured the Raman spectra of hydrogenated and deuterated biurea samples, in a DAC, carrying out more rapid compression than our neutron experiment.

Figure $5 \mathrm{~d}$ shows the Raman spectra of both perdeuterated and perhydrogenated biurea at ambient pressure. The mode assignment is based on that given in Ref. ? , and the observed vibrations agree well with those predicted by our periodic DFT calculations. We are confident that the samples are completely hydrogenated/deuterated, as there is a pronounced shift of the N$\mathrm{H} / \mathrm{D}$ stretching mode between 3250 and $2300 \mathrm{rel} \mathrm{cm}^{-1}$; change to the order of $\sim \sqrt{ } 2$ as expected. We observe a similar change in our simulated INS spectra from the DFT simulations, where the spectral range these modes cover shifts from $3100-3500 \mathrm{~cm}^{-1}$ to $2300-2600 \mathrm{~cm}^{-1}$ on deuteration and this is confirmed experimentally (Figure 5. and S.I. ${ }^{\dagger}$ ). The spectrum from the perdeuterated sample is intrinsically noisier compared to the perhydrogenated sample as a result of the significant difference in scattering cross section of deuterium (7.64 barn) compared to that of hydrogen (82.03 barn). The changes in Raman spectra with increasing pressure, measured from perdeuterated biurea between 80-220 rel cm ${ }^{-1}$, are shown in Figure 5 - - other spectral regions are available in the S.I. ${ }^{\dagger}$ As pressure is increased, reduced volume causes vibrational modes in both isotopologues to stiffen, shifting them to higher frequency. Across all spectral regions, we observe a clear change in compression trend around $0.49-0.58 \mathrm{GPa}$ and $0.72-0.78 \mathrm{GPa}$ for the deuterated and hydrogenated samples, respectively. While the shift in transition pressure is typical of isotopic substitution, ? they still occur over a relatively narrow range compared to the previous study.

Turning to the possible influence of compression rate, our use of the DAC, coupled with the rapid data acquisition rate of the Raman spectroscopy method, relative to neutron diffraction, resulted in much faster pressure increases. Plots of the individual modes as a function of pressure, shown in Figure 5 e and the S.I. ${ }^{\dagger}$, still indicate a relatively abrupt transition with the appearance of the majority of new modes appearing at the pressures marked by the dotted line, as well as discontinuities in modes that persist through the phase change. Thus we are unable to account for the difference between our study and that in Ref. ? .

\section{Conclusions}

We find that the high-pressure structure of biurea actually retains the same space group symmetry and topology as the ambientpressure phase. Although DFT calculations appear to indicate that the phase II structure should be the more stable, even under ambient conditions, only by considering the role of thermally influenced terms-in particular, ZPE and entropy in this casecan the stabilisation of phase I at ambient pressure be understood fully. Above ambient pressure, the need to minimise volume be- comes more important, favouring phase II, and overcoming the relatively diminished entropic contributions. This current work further demonstrates that vibrational contributions are important in isosymmetric transitions, where the change in atomic coordinates appears as though it could be achieved via displacement rather than an abrupt volume collapse. This phenomenon, rarely seen in organic compounds, ? may be more heavily dependent on vibrational free-energy stabilisation? than in other more reconstructive first order transitions.

\section{Conflicts of interest}

There are no conflicts to declare.

\section{Acknowledgements}

This work has been supported by facilities made available by STFC. We acknowledge ISIS Neutron and Muon Facility for access to the PEARL and TOSCA instruments? . We thank Dr Svemir Rudic, Dr Jeff Armstrong, and Dr Hamish Cavaye for assistance with the INS measurements and useful discussion. Finally, we acknowledge the computing resources provided the STFC Scientific Computing Department's SCARF cluster. 